\title{
Active Islanding Detection for Multiple Parallel- Connected Inverter-Based Distributed Generators Using High Frequency Signal Injection
}

\author{
David Reigosa, Fernando Briz, Cristian Blanco, Pablo García, Juan Manuel Guerrero \\ University of Oviedo. Dept. of Elect., Computer \& System Engineering, Gijón, 33204, Spain. \\ reigosa@isa.uniovi.es, fernando@isa.uniovi.es, $\underline{\text { blancocristian@uniovi.es, }}$ pgarcia@isa.uniovi.es, guerrero@isa.uniovi.es
}

\begin{abstract}
This paper proposes a method for islanding detection in microgrids with multiple parallel-connected inverters using high frequency signal injection. In the proposed method, a master inverter injects the high frequency signal which is used by the rest of inverters for islanding detection, with two distinguishing features: 1) The slave inverters work in a high frequency current cancellation mode, what prevents interference, and 2) in case of master failure or significant changes in the grid, the remaining inverters will dynamically reassign roles, the new master inverter being self-selected, based on a deterministic performance criteria and without the need of communications. ${ }^{1}$

Index Terms - Active islanding detection, grid impedance measurement, high frequency signal injection, power system monitoring, distributed generation.
\end{abstract}

\section{Introduction}

Distributed generation (DG) based on renewable and nonrenewable energy resources (photovoltaic, fuel cells, micro gas turbines, biomass, wind turbines, etc.) has been the focus of significant research efforts during the last years due to their increasing share in the generated energy. DG systems have to meet power quality requirements (e. g. harmonic distortion, EMI limits...) as well as power generation requirements. Among these, islanding detection is of special importance. Islanding is defined as the situation in which a distributed generator continues generating power when the microgrid is not connected to the utility grid. The requirements for islanding detection are regulated either by standardizing institutions as IEEE and IEC and/or by technical recommendations issued by different countries [112], examples of these being:

- The IEEE 1547 standard [1] requires the islanding detection in less than 2 seconds after the islanding condition occurs, which is the same as for IEC standard [6]. The same requirements are established by the Underwriters Laboratories Inc., specified in the UL 1741 standard [5], as well as by the IEEE 929 standard [4]. The German standard, DIN VDE 0126 [7] establishes that islanding detection methods should detect variations of the grid impedance of $1 \mathrm{Ohm}$ in 2 seconds.

\footnotetext{
1 This work was supported in part by the Research, Technological Development and Innovation Programs of the Ministry of Science and Innovation under grant MICINN-10-CSD2009-00046 and of the Spanish Ministry of Science and Innovation-ERDF under grants MICINN-10ENE2010-14941 and MICINN-10-IPT-370000-2010-15.
}

- The Austrian and Swiss standards [9, 10], establishes that islanding detection methods should detect variations of the grid impedance of $0.5 \mathrm{Ohm}$ in 5 seconds.

- The Australian standard [11] requires islanding detection within 2 seconds, as the IEEE 1547 standard.

Requirements on DPG often depend on their size and level of integration; low power DPG typically have to disconnect if the microgrid ceases energizing the distribution line, while larger power DPG may be requested to contribute to the stability of the grid. However, the future scenario might consider smart microgrids able to switch to stand-alone if faults occur in the distribution line, and then reconnecting to the grid [13].

Islanding detection methods can be classified into three groups:

- Passive methods: are inverter resident methods, in which changes in a power system parameter due to a power mismatch after disconnection is detected. They are normally easy and cheap to implement, being grid friendly as no additional signal is injected; however they present a large non-detection zone NDZ [12, 13].

- Communication methods: are highly flexible, having very low NDZ (no NDZ in theory). However, they are usually expensive due to the need of a communication infrastructure [12], also the risk of communication breakdown exists.

- Active methods: also inverter resident, in these methods a disturbance is generated, the grid response being measured. These methods have a low NDZ and are easier to implement than communication based methods. However, the can negatively impact the power quality, also interference among power converters trying to detect islanding simultaneously can occur $[12,13]$.

High frequency signal injection methods fall within active islanding detection methods category, appealing properties include a reduced Non-Detection-Zone (NDZ) and ease of implementation. However, interference between converters can occur if multiple inverters in a microgrid or in a grid have the capability of injecting the high frequency signal. One option to avoid interference between converters is to use a master-slave strategy, in which a master inverter is responsible of injecting the high frequency signal voltage, and the rest of (slave) inverters in the grid use this signal to 
detect islanding. Assignation of the master role can be done statically -a particular inverter is always the master- or dynamically -the master inverter is chosen among the inverters active in the network, based on some criteria, e.g. the proximity to the point of common coupling-. However, both solutions present problems. Failure of the master inverter for the case of a static assignment would leave the grid without the high frequency signal voltage, the rest of inverters in the grid hence loosing their capability to detect islanding. On the other hand, dynamic selection of the master inverter might require the use of communications, communication breakdown becoming therefore a concern.

This paper proposes a method for islanding detection using high frequency signal injection in grids with multiple parallel-connected inverters. In the proposed method, inverters dynamically respond to changes in the grid (e.g. failure of the master inverter, change from island to gridconnected or from grid-connected to island, ...) automatically reassigning the master-slave roles, without the need of communications. To coordinate the actions of the inverters present in the grid, the proposed method implements two distinguishing features:

1. The slave inverters work in a high frequency current cancellation mode. This mode of operation prevents the reaction of the slave inverters against the high frequency signal voltage injected by the master, still keeping full capability of fast and accurate islanding detection.

2. In the case of master failure, or significant changes in the grid, the remaining inverters will dynamically reassign roles, the new master inverter being self-selected based on a deterministic performance criteria and without the need of communication.

The paper is organized as follows: Section II presents a review of active islanding detection based methods; Section III presents the physical principles of the proposed islanding detection method based on high frequency current cancellation strategy, while Section IV presents the proposed method for dynamic master roll assignment. Discussion of implementation issues is presented in Section V. Finally experimental results to demonstrate the viability of the proposed method are presented in Section VI.

\section{Islanding detection using active methods.}

Active islanding detection methods measure the reaction of the grid to a small disturbance or test signal [1-12]. Active islanding detection methods can be divided in two groups:

\section{A) Grid variable variation methods}

In these methods, the DG produces small disturbance in a grid variable (e.g. voltage or frequency). The disturbance will normally have no measurable impact for the case of gridconnected operation, while it will be measurable for the case of island condition, the grid/islanding condition being therefore obtained from the grid/microgrid response [13].

B) Impedance estimation based methods
These methods can be divided into grid impedance estimation by active and reactive power variation [14] and methods using harmonic signal injection [15-19].

Active and reactive power variation based methods produce periodic variations of the converter output active and reactive power. This produces variations of the output current and voltage, from which the grid impedance is estimated [14].

Harmonic signal injection methods use some form of high frequency excitation and can be further classified into two groups:

- Intermittent signal injection based methods [15-18]. The signal is injected normally at the instant of zero crossing of the grid voltage, which implies that the islanding situation can only be detected at that instant of time. The frequency of the signal used in [15-17] was $75 \mathrm{~Hz}$ and in [18] 400$600 \mathrm{~Hz}$.

- Continuous signal injection based methods [19]. These methods allow almost instantaneous detection of the island/grid-connected condition [19], but this is achieved at the price of an increase of the THD [1-11]. In [19], a high frequency voltage as small as $0.3 \%$ of the line voltage, with a frequency of $300-400 \mathrm{~Hz}$, allowed reliable islanding detection, without compromising the THD restrictions.

Generally speaking, potential concerns for active methods are that they could negatively impact the power quality in grids with large penetration of inverter-based DG. In addition, interference problems among converters can occur if several DG's are connected in parallel to the same PCC, what might result eventually in an increase of the NDZ [13].

Fig. 1.- Generic microgrid architecture.

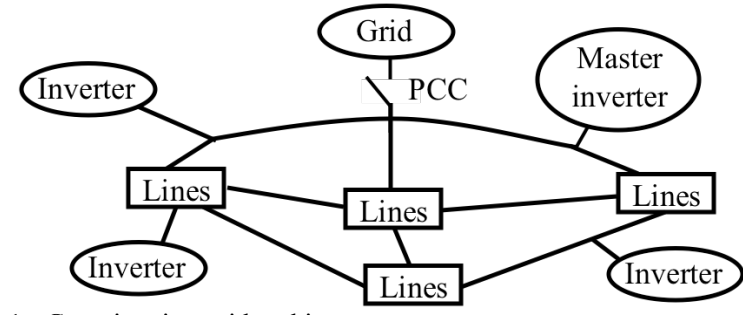

\section{Islanding detection using high frequency voltage injection and high frequency current cancellation}

It has already been shown that islanding detection in inverter-based DG can be done by measuring the grid response to a high frequency signal voltage [19]. While the implementation of this method is relatively simple when there is a master inverter responsible of injecting the high frequency signal (see Fig. 1), interferences can occur if multiple inverters connected in parallel do the same, strategies to prevent from this to happen being needed in this case $[15,19]$. The coordinated injection of a noncharacteristic harmonic $(75 \mathrm{~Hz})$ was proposed in [15], each inverter checking if this harmonic is present in the grid before injecting. When the harmonic is present, -i.e. other inverter 


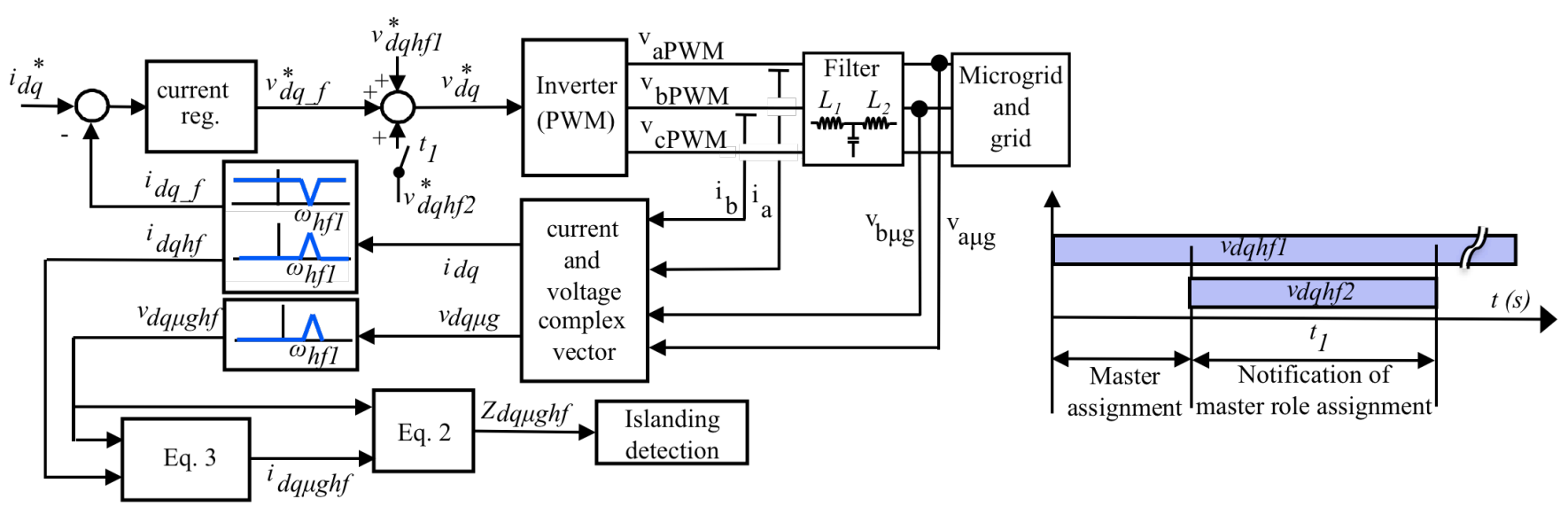

Fig. 2.- Control block diagram of the master inverter.

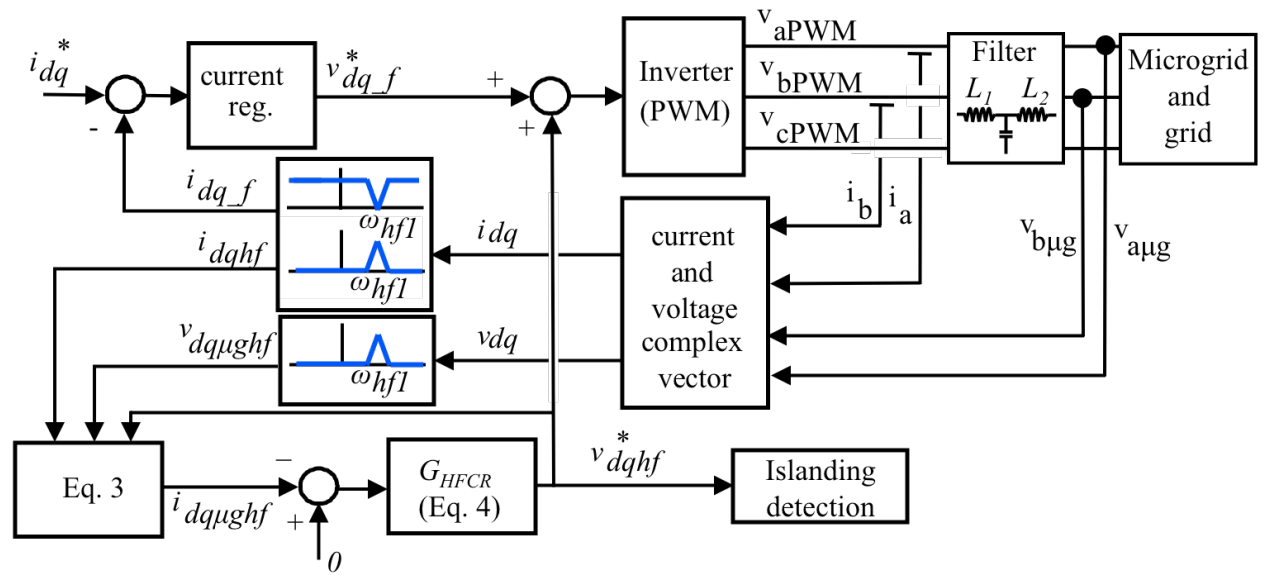

Fig. 3.- Control block diagram of the slave inverters.

is already injecting the signal-, the inverter waits a few cycles of the fundamental voltage before checking out again. On the other hand, if the harmonic is not present, the inverter will start injecting the signal and processing the resulting current to detect islanding. The reliability of this method is compromised when the number of inverters connected in parallel increases due to several reasons. The physical limit in the numbers of inverters connected is given by the number of zero crossing points during islanding detection (e.g. 240 zero crossing points during $2 \mathrm{sec}$. [1] in a $60 \mathrm{~Hz}$ grid), also interference between converters might still occur if several inverters start the check-and-inject process simultaneously $[15,19]$.

In [19], the use of a master inverter, responsible of injecting a high frequency rotating voltage vector (1), was proposed. Two high frequency signals are injected in this method. The first one is used for islanding detection, while the second one is used to communicate the islanding condition to the rest of inverters present in the grid. While no interference between inverters can occur -only the master inverter injects high frequency signals-, it has some limitations:

- Islanding detection capability is lost in case of master inverter failure.
- Current regulated VSI could react to (and partially compensate) the induced high frequency current, the particular effects of this reaction depending on the frequency of the injected signal and the bandwidth of the current regulators [19].

- The reliability of the proposed method could be compromised if the magnitude of the high frequency current/voltage becomes to small to be reliable detected by one or more microgrid inverters placed in critical points of the microgrid [19]. This will strongly depend on the microgrid architecture. Although strategies to prevent this are discussed in [19], it is at the price of an increase in the complexity of the method.

A strategy to overcome these limitations is proposed following. In the proposed method, the master inverter continuously injects the high frequency signal voltage $v_{\text {dqhf }}$ used for islanding detection (1) (see Fig. 2), the master inverter using a high frequency impedance variation criteria (2) to detect islanding [19]. The high frequency impedance is estimated using the measured LCL filter high frequency output voltage $v_{d q u g h f}$ and the estimated LCL filter high frequency output current (3).

$$
v_{d q h f 1}^{*}=V_{h f} e^{j \omega_{h f} t}
$$




$$
\begin{aligned}
& Z_{d q \mu g h f}=\frac{v_{d q \mu g h f}}{i_{d q \mu g h f}} \\
& i_{d q \mu g h f}=\frac{v_{d q h f 1}^{*}-v_{d q \mu g h f}-j \omega_{h f} L_{1} i_{d q h f}}{j \omega_{h f} L_{2}}
\end{aligned}
$$

where $\omega_{h f}$ is the frequency of the signal injected, $v_{d q h f 1}^{*}$ is the commanded high frequency voltage, $i_{\text {dqhf }}$ is the inverter induced high frequency current, $v_{d q u g h f}$ is the measured high frequency voltage at the filter output, $L_{1}$ is the inverter side inductance filter and $L_{2}$ is the microgrid side inductance filter. More details on the high frequency impedance estimation can be found in [19].

To prevent the reaction of slave inverters against the high frequency current, they include a PI current regulator in a reference frame synchronous with the injected high frequency voltage $\omega_{h f}$ (4). This current regulator sees the high frequency current at the inverter LCL filter output as a DC signal. By making its command equal to zero, it is guaranteed that no high frequency current exists at the inverter output (see Fig. 3).

$$
G_{H F C R}=k_{p}\left(1+K_{i} / s\right)
$$

This mode of operation for the slave inverters is called infinite impedance mode. In this mode of operation, slave inverters detect islanding/grid connected condition from the changes in the high frequency current regulator output voltage. This is described later in this section. It is noted that both the output current of the inverter and the output voltage of the LCL filter are normally available in inverter-based DG, no additional sensors are therefore needed to implement this method [19]. It is also important to notice that since no high frequency current exists at the slave inverters output, it will be the high frequency voltage command (i.e. the output of the high frequency current regulator in Fig. 3) the variable reflecting the grid connected/islanding condition.

This strategy has several appealing properties. The high frequency signal voltage injected by the master inverter is not corrupted due to the reaction of other inverters in the grid, as no high frequency current flows through them. This also prevents interference between inverters since only the master inverter injects the high frequency signal. It is finally noted that though the proposed strategy is described for current regulated VSI inverters using an LCL filter, it can also be implemented on inverters using other filter designs like inductive filters.

To evaluate the proposed method, a scenario with three parallel-connected converters -a master and two slave inverters-, has been built as an example (Fig. 4). The simulation parameters are summarized in Table I. Fig. 5 shows the simulation results. The master inverter continuously injects the high frequency voltage $\left(v_{\text {dqhf }}\right.$, see table I), the estimated magnitude and phase of the high frequency impedance being shown in Fig. 5a and $5 \mathrm{~b}$ respectively. Transition from island to grid-connected and form grid-connected to island occur at $\mathrm{t}=3.5 \mathrm{~s}$ and $\mathrm{t}=5.5 \mathrm{~s}$ respectively. It is observed from Fig. $5 \mathrm{a}$ and $5 \mathrm{~b}$ that the changes in the magnitude and phase of the high frequency impedance allow reliable island/grid-connected detection [19].

\begin{tabular}{|l|l|}
\hline \multicolumn{2}{|c|}{ Table I. Simulation parameters } \\
\hline Grid & $380 \mathrm{~V}, 50 \mathrm{~Hz}, \mathrm{~S}_{\mathrm{cc}}=15 \mathrm{MVA}$ \\
\hline Master inverter, inverter 1, and 2 & $380 \mathrm{~V}, 5 \mathrm{kHz}$. \\
\hline Load1,2,3 and 4 & $10 \mathrm{~kW}$ \\
\hline Line & $11.7 \mathrm{mOhm}, 8.68 \mathrm{e}-4 \mathrm{H}$ \\
\hline Simulation step & $1 \mathrm{e}-5 \mathrm{~s}$ \\
\hline$v_{\text {dqhf } 1}$ & $0.05 \mathrm{pu},-333 \mathrm{~Hz}$ \\
\hline$v_{\text {dqhf } 2}$ & $0.05 \mathrm{pu}, 275 \mathrm{~Hz}$ \\
\hline
\end{tabular}

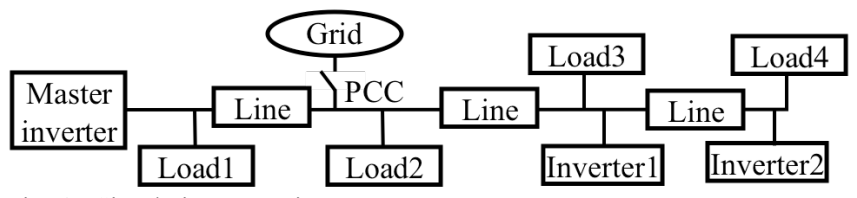

Fig. 4.- Simulation scenario.

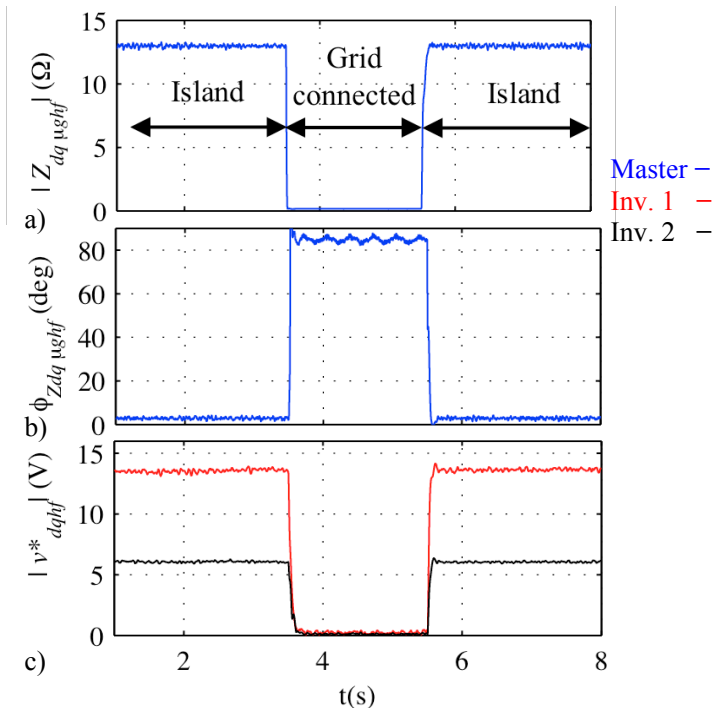

Fig. 5.- Simulation results showing a) the estimated high frequency impedance magnitude and b) phase, by the master inverter; c) inverter 1 and 2 high frequency current regulator output voltage magnitude.

Fig. 5c shows the magnitude of the high frequency current regulator output voltage $\left(\left|v_{d q h f}^{*}\right|\right.$, see Fig. 3$)$ for the two slave inverters (inverters 1 and 2 in Fig. 4). It can be observed that the high frequency voltage dramatically decreases when the microgrid becomes connected to the main grid. This is due to the variation of the high frequency impedance between island and grid connected condition. While in island condition the overall microgrid high frequency impedance corresponds to the local loads, and can be relatively large, in grid connected condition the overall high frequency impedance is dominated by the grid impedance, which is normally significantly smaller [13]. This high frequency impedance change produces a variation of the high frequency current regulator output voltage, which is used by the slave inverters to detect the islanding/grid-connected condition. This is done without 


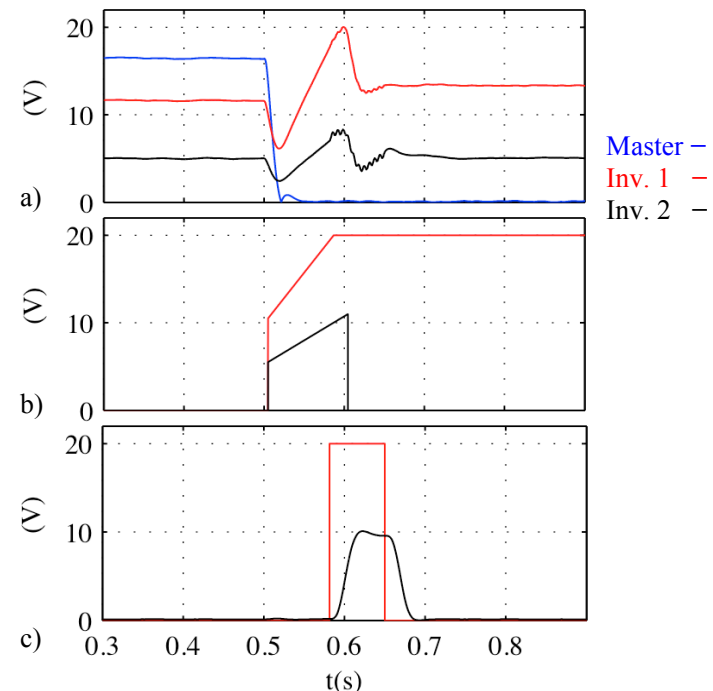

Fig. 6.- Simulation results showing the high frequency voltage magnitude measured at the LCL filter output, for the master inverter, inverter 1 and inverter 2, a), commanded high frequency voltage magnitude of inverter 1 and 2 after detecting the high frequency voltage lost, b), and magnitude of the secondary high frequency voltage injected by inverter 1 after assuming the master role, and measured by inverter $2, \mathrm{c}) . V_{h f l}=0.05 \mathrm{pu}$, $\omega_{h f 1}=-333 \mathrm{~Hz}, V_{h f 2}=0.05 \mathrm{pu}, \omega_{h f 2}=303 \mathrm{~Hz}$.

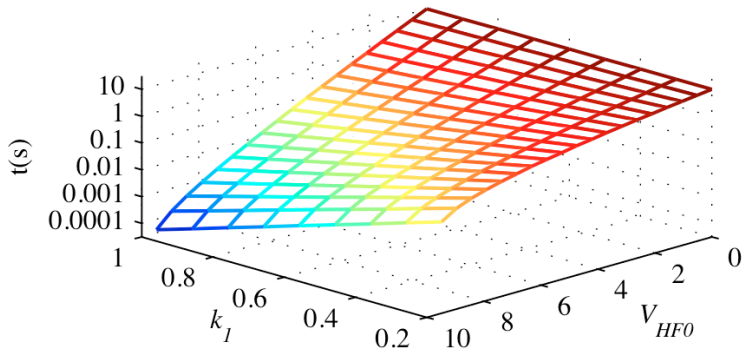

Fig. 7.- Elapsed time to reach the rated high frequency voltage magnitude as a function of the initial condition $V_{H F 0}$ and of gain $k_{1}$, with gain $k_{2}=1$.

the need of a secondary high frequency signal for communication purposes [19] and without interfering with the master inverter operation.

It is noted that a threshold for the high frequency current regulator output voltage magnitude needs to be set to detect the transition between islanding and grid-connected operation. This threshold might depend on the microgrid/grid architectures, microgrid/grid high frequency impedances or grid pollution. As for the islanding detection THD based methods $[13,19,20]$ or the voltage harmonic monitoring based methods [21], the selection of the threshold is not always easy and might require a previous knowledge of the microgrid/grid characteristic.

\section{Dynamic master role assignment}

One concern of methods in which a master inverter is responsible on injecting the high frequency voltage, is the risk of a master inverter failure [19]. This will leave the $\mathrm{grid} /$ microgrid without the high frequency signal, the slave inverters therefore loosing their islanding detection capability. Further more, even in the event that the master inverter does not fail, changes in the grid/microgrid topology, e.g. switching between islanding and grid connected operation, connection/disconnection of other inverters, generation units, loads, etc, might have a significant impact in the high frequency signal seen by the inverters connected to the microgrid, eventually affecting to the performance of the method. E.g. during grid-connected operation several inverters might be working like masters, due to the low grid impedance, while during islanding, it is likely that one single master inverter might be enough to supply a high frequency signal voltage visible to all the inverters connected to the microgrid. Hence, in the event of switching from grid connected to islanding, all the master inverters but one could likely safely switch to the slave mode. It is concluded that some mechanism for the dynamic reassignment of the master/slave roles is convenient. Using communications or some type or pre-configuration of the master/slave inverters for this purpose is considered unappealing. A strategy for the dynamic reassignment of the master role is proposed in this paper. It is accomplished in three steps.

1. Detection by the inverters of changes in the microgrid. Such changes can be of three types. a) the master inverter detects a sudden variation of the high frequency impedance; b) the slave inverters detect that no high frequency voltage is present in the grid. This can be due either to the failure of the master inverter, or to a significant decrease of the grid impedance (e.g. due to a change from island to grid connected operation); and c) the slave inverters detect a sudden increase of the high frequency voltage in the microgrid. This can be due to a change from grid connected to islanding. An example of failure of the master inverter is shown in Fig. 6 (the grid topology is shown in Fig. 4). Fig. 6a shows the high frequency voltage magnitude, measured at the LCL filter output, for the master power converter (blue) and two slave power converters, master failure occurring at $\mathrm{t}=0.5 \mathrm{~s}$

2. Reassignment of the master inverter role. Once any of the events described above occurs, all the inverters start injecting the high frequency voltage immediately. However, the slave inverters do not inject a constant magnitude high frequency voltage, it increases with time instead (5)-(6), where $V_{H F 0}$ is the magnitude of the high frequency voltage for each slave inverter right before the master inverter failure, and $V_{H F_{-} \max }$ is the limit established for the high frequency voltage.

$$
\begin{array}{ll}
v_{H F}=\left(V_{H F 0}+k_{2} t e^{k_{1} V_{H F 0}}\right) e^{j \omega_{h f} t} & \text { if }\left|v_{H F}\right|<V_{H F_{-} \max } \\
v_{H F}=V_{H F_{-} \max } e^{j \omega_{h f} t} & \text { if }\left|v_{H F}\right| \geq V_{H F_{-} \max }
\end{array}
$$

It is observed from (5) that the high frequency voltage magnitude increases linearly with time, but both the starting value as well as the rate of increase being function of $V_{H F 0}$. According to (5), the inverter with a larger initial voltage $V_{H F 0}$ starts with a larger initial value and increases the magnitude faster (see Fig. 6b), 
therefore reaching $V_{H_{-} \text {max }}$ first. The philosophy behind this strategy is that, in general, inverters closer to the PCC before the failure are considered better candidates to assume the master role. The strategy established by (5)(6) will produce that the inverters placed closer to the PCC will win the race to become master.

Fig. 7 shows the time needed by an inverter to reach $V_{H F_{\text {max }}}$, as a function of $V_{H F 0}$ and the gain $k_{1}$. It is observed from the figure that small differences in $V_{H F 0}$ produce large variations in the time needed to reach $V_{H F_{-} \max }$, therefore reducing the risk of more than one inverter becoming master at the same time.

Once one inverter reaches $V_{H F_{-} \text {max }}$, i.e. the rated high frequency voltage magnitude, automatically changes its role to master (Fig. 2) and continues injecting the high frequency signal hereafter (see Fig. 6b).

It is noted that other reassignment strategies different from (5)-(6) could be used, the key issue for the design of new strategies being their ability to discriminate the most suitable inverters to assume the master role during the master reassignment process. It is also interesting to note that although in the strategy used in this work the high frequency signals are injected during the reassignment process (see Fig. 6), it is feasible for the inverters to produce the high frequency signal internally, i.e. without physically injecting them intro the grid, in such a way that only when a inverter reaches $V_{H F_{-} \max }$ it becomes the master inverter, and injection of the actual high frequency voltage into the grid occurs.

3. Notification of the role change to the rest of microgrid inverters. Once one inverter assumes the master role, it injects a secondary high frequency signal $\left(v_{d q h f 2}\right)$ during a short period (80ms, see Fig. 6c). This signal informs to the rest of inverters that the master role has already been reassigned. The rest of inverters automatically will stop injecting the high frequency signal and change their mode of operation to slave mode (Fig. 3). Fig. 6c shows the commanded voltage magnitude of the secondary high frequency signal by inverter 1 and the measured secondary high frequency voltage magnitude by inverter 2, which is used to stop its high frequency signal injection (see Fig. 6b) and to switch to the slave mode.

\section{High frequency signal selection}

Both the magnitude $\left(V_{h f}\right)$ and the frequency $\left(\omega_{h f}\right)$ of the high frequency voltage injected by the master inverter need to be set. Generally speaking, low-magnitude, high frequency signals will decreases the adverse impact on the THD, what needs to be considered to meet the connection standards [1$11]$.

A relatively large frequency range could be used, the limits coming from the interaction with the output LCL filter resonant frequency (see Fig. 2 and 3), the grid resonant frequency and the need of having spectral separation with fundamental frequency dependent harmonics (e.g. $-5^{\text {th }}, 7^{\text {th }} \ldots$ ) that often exist [19]. It is noted in this regard that the injected high frequency signal can have a positive or negative frequency, as it is complex vector. The high frequency signal can be chosen to be synchronous (i.e. an integer multiple like $\left.\pm 6^{\text {th }},+5^{\text {th }},-7^{\text {th }} \ldots\right)$ or asynchronous with the fundamental frequency. Being in synchronism might provide some advantages, e.g. during the reassignment of the master role, all the inverters could inject the high frequency signal in phase with each other, the process being then more repetitive. However, synchronizing the high frequency signal with the fundamental voltage requires the estimation of the fundamental voltage phase angle, which might be problematic in the case of distorted grids. An asynchronous high frequency signal with $\omega_{h f}=-333 \mathrm{~Hz}$ was used for the simulation and experimental results shown in this paper (see Table I).

\begin{tabular}{|l|l|}
\hline \multicolumn{2}{|c|}{ Table II. Experimental setup parameters } \\
\hline Grid & $380 \mathrm{~V}, 50 \mathrm{~Hz}, \mathrm{~S}_{\mathrm{cc}}=2 \mathrm{MVA}$ \\
\hline Generator $(\mathrm{G})$ & $100 \mathrm{kVA}, 380 \mathrm{~V}, 152 \mathrm{~A}$ \\
\hline Master inverter, inverter 2 and 3 & $380 \mathrm{~V}, 30 \mathrm{kVA}, 10 \mathrm{kHz}$. \\
\hline Load & $15 \mathrm{~kW}$ \\
\hline$v_{\text {dqhf1 }}$ & $0.03 \mathrm{pu},-333 \mathrm{~Hz}$ \\
\hline$v_{\text {dqhf } 2}$ & $0.03 \mathrm{pu}, 303 \mathrm{~Hz}$ \\
\hline LPF bandwidth & $25 \mathrm{~Hz}$ \\
\hline HPF bandwidth & $25 \mathrm{~Hz}$ \\
\hline LCL filter resonance frequency & $575 \mathrm{~Hz}$ \\
\hline
\end{tabular}

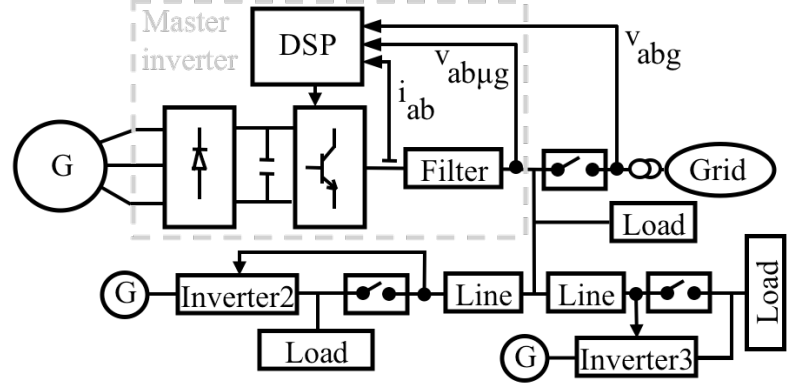

Fig. 8.- Experimental setup.

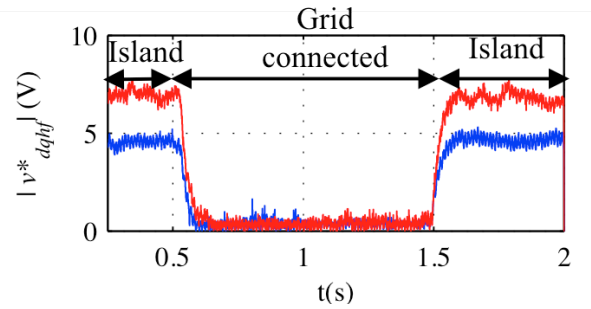

Fig. 9.- Experimentally measured magnitude of the high frequency current regulator output voltage of inverters 2 (red) and 3 (blue) for island/grid connected operation. The master inverter injected a high frequency voltage of $V_{h f l}=0.03 \mathrm{pu}, \omega_{h f l}=-333 \mathrm{~Hz}$.

The magnitude of the injected high frequency signal in the simulation results shown in this paper was set to $0.05 \mathrm{pu}$ of the line-to-line voltage. The THD when the high frequency signal is not injected is $\approx 1.15 \%$ while it increases to $\approx 1.38 \%$ when the high frequency signal was injected, not compromising therefore the connection standards [1-11]. 
For the secondary high frequency signal $\left(v_{d q h f 2}\right)$, which is injected only for communication purpose, the magnitude should be high enough to enable reliable detection by the remaining microgrid inverters, while the same considerations as for $v_{\text {dqhf } 1}$ could be made for its frequency. A magnitude of $0.05 \mathrm{pu}$ was found enough for reliable reception by the remaining microgrid inverters.

\section{Experimental results}

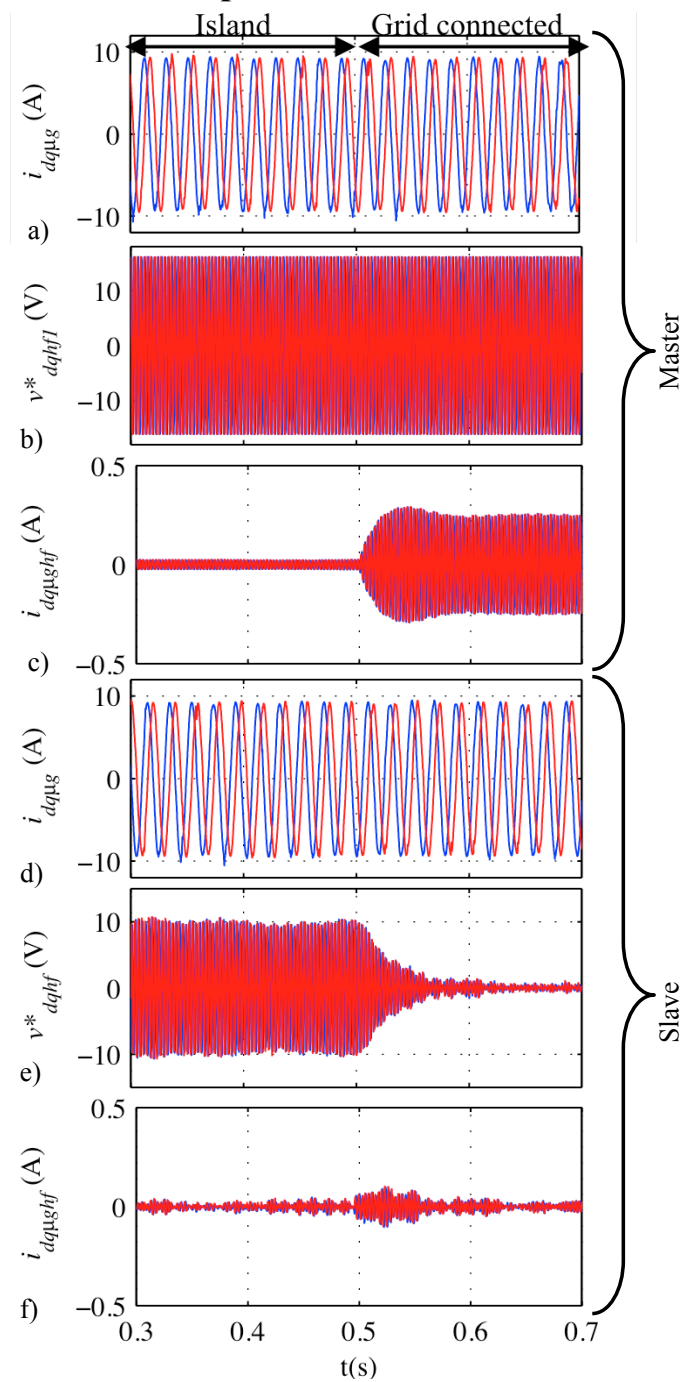

Fig. 10.- Experimental results showing the transition from island to gridconnected situation ( $\mathrm{t}=0.5 \mathrm{~s}$ ). a) to c) Master inverter: a) $d$ and $q$-axis components of the measured output LCL filter current, b) injected high frequency voltage by the master inverter, c) $d$ and $q$-axis components of the measured output LCL filter high frequency current (obtained by digital signal processing of the overall filter current); d) to f) slave inverter (\# 2): d) $d$ and $q$-axis components of the measured output LCL filter current, e) injected high frequency voltage by the slave inverter (current regulator reaction) and $\mathrm{f}$ ) $d$ and $q$-axis components of the measured output LCL high frequency current. $V_{h f 1}=0.03 \mathrm{pu}, \omega_{h f 1}=-333 \mathrm{~Hz}$.

To verify the viability of the proposed islanding detection and dynamic master assignment methods, the experimental setup shown at Fig. 8 was used. Though not shown in the figure, inverters 2 and 3 have the same topology as the master inverter, each including a DSP TMS320F28335 for their control. The parameters of the experimental setup are summarized in Table II. All the inverters include voltage sensors in the line side, which are needed for synchronization. The computational requirements for the implementation of the method are of $8.2 \mu$ s for the master inverter operation mode and $12.8 \mu$ s for the slave inverter operation mode

Fig. 9 shows the magnitude of the output voltage of the high frequency current regulator (4) of inverters 2 and 3 during transitions from island to grid connected $(\mathrm{t}=0.5 \mathrm{~s})$ and back to island $(\mathrm{t}=1.5 \mathrm{~s})$. The master inverter was injecting the high frequency voltage continuously. The magnitude of the high frequency current regulator output voltage reliably reflects the transitions between island and grid connected, the changes in the operating condition being detected in tens of $\mathrm{ms}$, which is in compliance with international standards requirements [1-11].

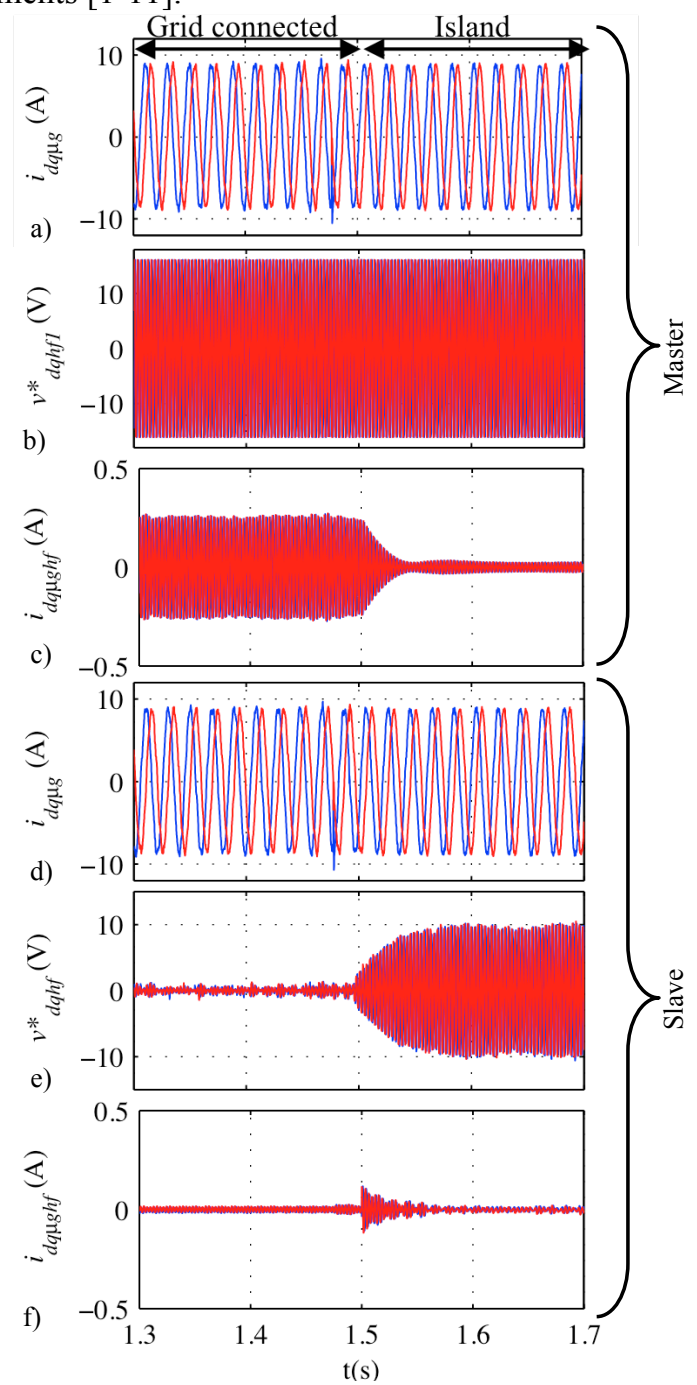

Fig. 11.- Same variables an operating condition as Fig. 10 during a transition from island to grid-connected situation. 
Fig. 10 shows the transient response of the master inverter and inverter 2 during a transition from island to gridconnected. Fig. 10a shows the $d$ and $q$-axis components of the measured output current of the master inverter LCL filter. Fig. 10b shows the injected high frequency voltage, while Fig. 10c shows the resulting high frequency current. It is observed that the master inverter is continuously injecting a high frequency signal, the resulting current strongly changing from island to line connected, which is used by the master inverter to detect the islanding/grid connected situations as described in [19]. Fig $10 \mathrm{~d}$ shows the $d$ and $q$-axis components of the current measured at the slave inverter LCL filter, while Fig. 10e and 10f shows the high frequency voltage injected by the slave inverter as well as the high frequency current at the slave inverter output. The infinite impedance mode of operation describe previously causes the high frequency current to be equal to zero in steady state.

Fig 11 shows the same results as Fig. 10 during a transition for grid connected to island situation, a similar behavior of the measured LCL filter output current is observed.

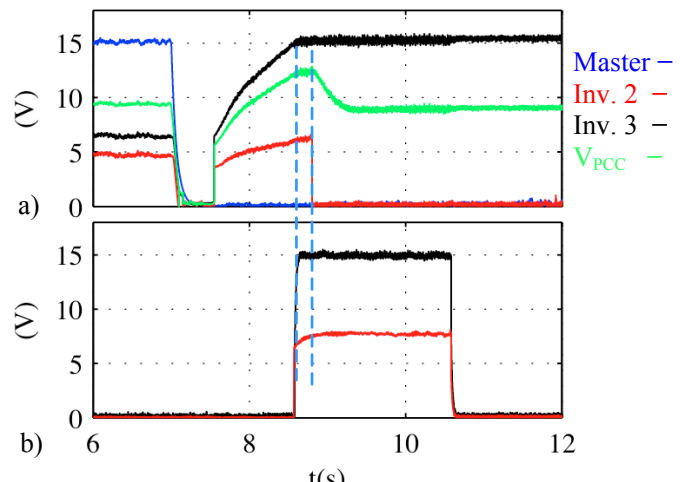

Fig. 12.- a) Magnitude of the high frequency voltage injected by the master inverter, inverters 2 and 3 and high frequency voltage magnitude at the PCC, when the failure of the master inverter occurs, and b) magnitude of the secondary high frequency voltage injected by inverter 2 and measured by inverter 3 after inverter 2 assumes the master role. $V_{h f l}=0.03 \mathrm{pu}, \omega_{h f l}=-333 \mathrm{~Hz}, V_{h f 2}=0.03 \mathrm{pu}, \omega_{h f z}=303 \mathrm{~Hz}$. The dotted lines indicate the instant in which inverter 2 assumes the master role (left) and inverter 3 detects the secondary high frequency signal and assumes the slave role (right).

Fig 12 a shows the dynamic reassignment of the master role after the failure of the master inverter (occurs at $t=7 \mathrm{~s}$ in Fig. 12). It is observed from Fig. 12a that after the master inverter failure detection, inverter 2 and 3 start injecting the high frequency signal as described by (5) and (6). Once inverter 2 reaches the rated high frequency voltage (see Fig. 12a), automatically changes its role to master (see Fig. 2), continues injecting the high frequency signal, and injects a burst with the secondary high frequency signal to indicate to the rest of the inverters that the master role has already been reassigned (see Fig. 12b). Fig. 12b shows the magnitude of the injected secondary high frequency voltage by inverter 2 (new master) and measured by inverter 3. It is observed in Fig. $12 \mathrm{~b}$ that inverter 3 , stops injecting the high frequency signal (red) immediately once it detects the presence of the secondary high frequency signal $\left(v_{d q h f 2}\right)$. It is also noted that although $v_{d q h f 2}$ is injected during 2 seconds, inverter 1 detected the presence of the secondary signal in less than $100 \mathrm{~ms}$, thus the injection time could be reduced.

It is finally noted that the THD at the PCC is only slightly affected by the injection of the different high frequency voltages, increasing from $2.13 \%$ for the case when no high frequency signal voltage is injected, to $2.87 \%$ when the high frequency signals for both islanding detection and communications are injected.

\section{Conclusions}

An active method to detect islanding in grids with multiple parallel-connected inverters has been presented in this paper. In the proposed method, one inverter (master) continuously injects a high frequency signal for islanding detection, while the rest (slave) inverters use a high frequency current cancellation strategy to detect islanding. By doing this, interference between converters is avoided, as no high frequency current circulates to/from the slave inverters.

A strategy for dynamic reassignment of the inverters role (master/slave), without the need of communications or preconfigured roles has also been proposed. The reassignment is done in three steps: 1) detection of significant changes in the grid, including master inverter failure and gird/island transitions, 2) reassignment of the master inverter role and 3) notification to the rest of microgrid inverters.

Simulation and experimental results have been presented to demonstrate the operation of both concepts.

\section{VIII.References.}

[1] IEEE, "IEEE Standard for Interconnecting distributed resources with electric power systems," IEEE Std. 1547, 2003.

[2] IEC, "IEC Photovoltaic (PV) systems. Characteristics of the utility interface," IEC Std. 61 727, 2004.

[3] ENEL, "DK 5940 Criteria for connection of generation systems to ENEL low voltage distribution networks," 2006.

[4] IEEE Std. 929-2000, IEEE Recommended Practice for Utility Interface of Photovoltaic (PV) Systems, IEEE Standards Coordinating Committee 21 on Photovoltaics, New York, NY, Apr. 2000.

[5] UL1741, UL Standard for Safety for Static Converters and Charge Controllers for Use in Photovoltaic Power Systems, Underwriters Laboratories, May 7, 1999, revised June 2001.

[6] IEC 62116, Testing Procedure of Islanding Prevention Measures for Grid Connected Photovoltaic Power Generation Systems, International Electrotechnical Commission.

[7] DIN-VDE, "Automatic Disconnection Device Between a Generator and the Low-Voltage Grid," DIN-VDE Std. 0126-1-1, 2005.

[8] G77 - Recommendations for the Connection of Inverter- Connected Single-Phase Photovoltaic (PV) Generators up to 5kVA to Public Distribution Networks.

[9] ÖVE/Önorm E 2750 "Photovoltaische Energieerzeugungsanlagen Sicherheitsanforderungen ("Photovoltaic power generating systems safety requirements").

[10] VSE Sonderdruck Abschnitt 12 'Werkvorschriften über die Erstellung von elektr. Installation' Elektrische Energieerzeugungsanlagen Completes VSE 2.8d-95.

[11] Australian Standard AS4777, "Grid Connection Of Energy Systems Via Inverters Part 3: Grid Protection Requirements".

[12] A. Timbus, A. Oudalov, C. N.M. Ho, "Islanding Detection in Smart Grids," IEEE-ECCE'10, pp.3631 - 3637, Sep. 2010.

[13] R. Teodorescu, M. Liserre, P. Rodriguez and F. Blaabjerg, Grid Converters for Photovoltaic and Wind Power Systems, Wiley-IEEE 2011. 
[14] M. Ciobotaru, R. Teodorescu, P. Rodriguez, A. Timbus and F. Blaabjerg, "On-line Grid Impedance Estimation for Single Phase GridConnected Systems Using PQ Variations", IEEE-PESC, pp.2306-2312, June 2007.

[15] A. V. Timbus, R. Teodorescu and U. Borup, "Online Grid Impedance Measurement Suitable for Multiple PV Inverters Running in Parallel", IEEE-APEC'06, pp.907-911, March 2006.

[16] L. Asiminoaei, R. Teodorescu, F. Blaabjerg and U. Borup, "A Digital Controlled PV-Inverter with Grid Impedance Estimation for ENS Detection”, IEEE Trans. on Power Elect., 20(6):1480-1490, Nov.-Dec. 2005.

[17] L. Asiminoaei, R. Teodorescu, F. Blaabjerg and U. Borup, "A New Method of On-Line Grid Impedance Estimation for PV Inverter", IEEE-APEC'04, pp.1527-1533, Sept. 2004.

[18] M. Ciobotaru, R. Teodorescu and F. Blaabjerg, "On-line Grid Impedance Estimation Based on Harmonic Injection for GridConnected PV Inverter", IEEE-ISIE, pp.2473-2442, June 2007.

[19] D. Reigosa, F. Briz, C. Blanco, P. Garcia and J. M. Guerrero, "Active Islanding Detection Using High Frequency Signal Injection", IEEE Trans. on Ind. Appl., 48(5): 1588-1597, Sept./Oct. 2012.

[20] D. N. Gaonka, "Distributed Generation", In-Tech 2010.

[21] S. I. Jang and K. H. Kim, "An Islanding Detection Method for Distributed Generation Using Unbalance and Total Harmonic Distortion of Current", IEEE Trans. on Power Elect., 19(2):745-752, Apr. 2004. 